\title{
Dual-world hardware and software optics educational system
}

Preben Buchhave, Nini Pryds, Haim Abitan, Peter Tidemand-Lichtenberg

Preben Buchhave, Nini Pryds, Haim Abitan, Peter Tidemand-Lichtenberg, "Dual-world hardware and software optics educational system," Proc. SPIE 9664, Ninth International Topical Meeting on Education and Training in Optics and Photonics, 96641H (24 October 2005); doi: 10.1117/12.2207730

Event: Ninth International Topical Meeting on Education and Training in Optics and Photonics, 2005, Marseille, France 


\title{
Ref ETOP067
}

\section{Dual-world hardware and software optics educational system}

\author{
Preben Buchhave, Nini Pryds, Haim Abitan, and Peter Tidemand-Lichtenberg
}

\begin{abstract}
We have developed an educational optics learning tool, which allows students to work in parallel with a real hardware system and a matching, realistic looking, virtual model. We have implemented the idea as an educational laser-kit, and we describe the educational ideas behind it and our experience with its use for motivating and inspiring grade school teachers, educate high school students and train college students. The present system is a relatively advanced laser system consisting of a unique combination of a 2-D breadboard with magnetic mounts carrying the optical components and a corresponding, realistic 2-D display, layout and analysis software.
\end{abstract}

\section{Introduction}

\section{Summary}

We have found that laser design and experimentation tools do not take enough advantage of the possibilities offered by modern computer display and graphics capabilities. Consequently, we have experimented with a unique combination of a 2-dimensional magnetic breadboard (2-dimensional meaning with the possibility of placing components freely anywhere on a plane surface) and a corresponding realistic graphic display of the same setup (a virtual breadboard with virtual components). This unique, realistic correspondence allows user to move back and forth between the virtual setup, where he can analyze the layout and the beam properties, and the real breadboard, where he can place the components at the coordinates found from the software analysis. The precise one-to-one correspondence between hardware and software helps scientists and engineers make better and faster design.

The goal of our development efforts has been to create an efficient tool for experimentation with setups using laser beams, for design and prototyping of lasers and nonlinear optics and for work with other laser based applications.

\section{System description}

In the present phase the educational system consists of a standing wave laser based on a Nd:YVO4 crystal and a semiconductor pump laser. The system also allows intracavity second harmonic generation and pulsed operation with an intracavity saturable absorber. The students can place the components on the breadboard and experiment with the laser properties such as alignment, stability of the resonator, measurement of laser characteristic etc. while simultaneously examining the laser system theoretically by experimenting with the virtual model. In the hardware, the students can insert and remove components and slide them freely over the surface of the breadboard to test different configurations. Simultaneously, the students can try the same configurations in the virtual model and compare the model predictions to the actual behaviour of the laser. The software displays a natural looking optical baseplate with realistic renderings of the mounted components. For more advanced stud ents, the system can demonstrate concepts from nonlinear optics, Qswitched operation and mode matching as we find it for example in external cavity nonlinear conversion and in the case of coupling of two optical resonators. 


\section{Educational results}

We have tested the system in a wide range of educational settings ranging from grade school science teaching to university labs. Our experience is that we can present the idea of a laser successfully in all these environments by a preparing a suitable pedagogical description. The unique breadboard with magnetic mounts that can be placed freely and moved around on the breadboard surface makes it very fast and easy to set up and align the laser. The students have also been quick to learn to use the software and to grasp the idea of a "dual world" where they can compare the real laser to the software simulation. The result is a significant reduction set up time and time used for experimentation. We believe we have developed a unique system, which really takes advantage of the extra motivation and understanding that results from a good theoretical understanding and a corresponding "hands-on" experience with the real world. 